J. Clin. Chem. Clin. Biochem.

Vol. 19, 1981, pp. 1013-1019

\title{
Cholesterin und Triglyceride im Serum einer Münchner Bevölkerungsgruppe: Beziehungen zu Alter und Geschlecht ${ }^{1}$ )
}

\author{
Von K. Henze, Agnes Wallmüller-Strycker, M. Bauer, C. Barth, G. Wolfram und N. Zöllner \\ Forschergruppe Ernährung an der Medizinischen Poliklinik der Universität München
}

(Eingegangen am 6. August 1980/19. Februar 1981)

\begin{abstract}
Zusammenfassung: Bei 814 Personen wurden die Konzentrationen von Cholesterin und Triglyceriden im Serum bestimmt und auf ihre Abhängigkeit von Alter, Geschlecht und Körpergewicht untersucht. Bei 568 Personen wurden der BMI (body mass index) sowie das Relativgewicht nach Broca berechnet. Es wurden Referenzbereiche, nach Alter und Geschlecht getrennt, ermittelt. Cholesterin, Triglyceride und BMI stiegen bei Männern und Frauen mit dem Alter an. Eine positive Korrelation bestand zwischen BMI und den Triglyceridwerten, jedoch nicht zwischen BMI und Cholesterinwerten. Die Korrelation von BMI und Triglyceridwerten erwies sich als unabhängig vom Alter.
\end{abstract}

Die vorgelegten Daten bekräftigen den Zusammenhang zwischen Ubergewicht und Hypertriglyceridämie.

Serum cholesterol and triglyceride levels in a Munich population group: Relations to age and sex

Summary: Serum cholesterol and triglyceride levels were determined in 814 subjects and the relation to age, sex and body weight evaluated. In 568 persons the body mass index (BMI) and the relative body weight according to Broca were calculated. Normal values for each age group were established. Serum cholesterol, serum triglycerides and the body mass index showed an age dependent rise in the male and the female population. The body mass index showed a positive correlation with the serum triglycerides, but did not correlate with the serum cholesterol levels. The correlation between body mass index and serum triglycerides was not age dependent.

Thus, our data stress the relation between overweight and hypertriglyceridaemia.

\section{Einführung}

Es gibt zahlreiche Hinweise dafür, daß erhöhten Blutfettkonżentrationen eine große Bedeutung in der Pathogenese der Atherosklerose, speziell der großen Gefäße und der Coronararterien zukommt. In epidemiologischen Untersuchungen wurde $\bar{z}$. B. eine enge Beziehung zwischen Cholesterinkonzentrationen im Serum und der Mortălität àn coronarer Herzkrankheit nachgewiesen $(1,2)$. Auch die Triglyceridkonzentrationen im Serum könnten in dieser Beziehung von Bedeutung sein $(3,4)$. $\mathrm{Ob}$ Übergewicht als ein Risikofaktor für die coronare Herzkrankheit anzusehen ist, scheint ungewiß (5), doch wurden in einigen Untersuchungen Hinweise dafür gefunden (6).

Darübè hinaus zeigten experimentelle Untersuchungen am Menschenaffen, daß man durch eine diätetische und pharmakologische Senkung der Cholesterinwerte eine Rückbildung artherosklerotischer Läsionen herbeiführen

1) Mit Unterstützung der Deutschen Forschungsgemeinschaft. kann (7). Von anderen Autoren konnten Hinweise dafür gefunden werden, daß auch beim Menschen die Senkung der Cholesterinkonzentrationen mit einer Regression von Gefäßveränderungen und deren Folgen einhergeht (8).

Die Prävention und Therapie der coronaren Herzkrankheit stellt eine dringliche Aufgabe dar. Eine notwendige Voraussetzung für Diagnose und Behandlung des wichtigen Risikofaktors Hyperlipidämie ist die Ermittlung von Referenzbereichen für Lipide im Serum. Wir werden Referenzbereiche für Cholesterin und Triglyceride in Abhängigkeit von Alter und Geschlecht mitteilen; ihre Beziehung zum Übergewicht wird diskutiert.

\section{Methodik}

Insgesamt wurde das Serum von 814 Personen einer Münchner Bevölkerungsgruppe untersucht, die sich aus 580 Arbeitern und Angestellten, zum Teil schon pensionierten Mitarbeitern zweier Münchner Industriebetriebe sowie 234 Blutspendern des Gesundheitsamtes der Stadt München zusammensetzte. Die Alters- und Geschlechtsverteilung des Gesamtkollektivs 
ist der Tabelle 1 zu entnehmen. Die Blutabnahmen erfolgten anläßlich einer betriebsärztlichen Kontrolle bzw. einer freiwilligen Blutspende. Für die Untersuchungen wurde nur das Blut derjenigen Personen verwendet, die das 20. Lebensjahr vollendet hatten und die erklärten, vor mehr als zehn Stunden ihre letzte Mahlzeit zu sich genommen zu haben. $10 \mathrm{ml}$ Blut wurden in ein Glasröhrchen abgenommen, nach Eintritt der Gerinnung zentrifugiert, und das Serum bis zur Durchführung der Analysen bei $-20^{\circ} \mathrm{C}$ aufbewahrt. Von allen Personen wurde das Geburtsdatum, von einem großen Teil $(n=568)$ auch das Körpergewicht und die Körperlänge ermittelt. Die Cholesterinbestimmung erfolgte mittels einer vollenzymatischkolorimetrischen Methode nach Röschlau et al. $\left.(9)^{2}\right)$, die der Trigly ceride unter Verwendung einer Modifikation ${ }^{3}$ ) der Methode von Eggstein \& Kreutz (10). Da eine vertretbare Übereinstimmung zwischen Gesamtglycerin und Glyceridglycerin besteht, wurde freies Glycerin weder durch Messung noch durch Berechnung berücksichtigt (10). Die Hydrolyse der Triglyceride erfolgte durch Lipase. Die Qualitätskontrolle wurde mit Precilip ${ }^{\circledR}$ der Firma Boehringer GmbH, Mannheim, durchgeführt. Die Analysen wurden im Stoff wechsellabor unserer Klinik am Eppendorf Substratmeßplatz 5091 als Zweifachbestimmungen durchgeführt. Der Variationskoeffizient war bei der Cholesterinbestimmung $<3$, bei der Triglyceridbestimmung $<4 \%$.

Zur Prüfung einiger Korrelationen war es notwendig, die ermittelten Lipidwerte nach Alter und Geschlecht zu korrigieren. Im Alter von 45 Jahren lagen die Cholesterin-, im Alter von 55 Jahren die Triglyceridwerte bei Männern und Frauen auf gleicher Höhe. Der korrigierte Wert wurde mit Hilfe folgender Formel ermittelt:

$$
\mathrm{X}_{\text {korr. }}=\mathrm{X}_{\mathrm{E}}+\left(\mathrm{X}_{\mathrm{A}}-\mathrm{X}_{\mathrm{AG}}\right)
$$

Der korrigierte Wert $\left(X_{\text {korr }}\right)$ ergibt sich aus dem gemessenen einzelnen Wert $\left(X_{E}\right)$ plus der Differenz zwischen dem Mittelwert in der Altersklasse 45 (für Cholesterin) bzw. 55 (für Triglyceride) $\left(X_{A}\right)$ und dem Mittelwert des gemessenen Parameters in der entsprechenden Alters- und Geschlechtsklasse $\left(X_{A G}\right)$.

2) Test-Kombination, Boehringer GmbH, Mannheim, Nr. 124087.

3) Test-Kombination, Boehringer GmbH, Mannheim, Nr. 126039.
Abweichungen vom Sollgewicht wurden mit Hilfe des BodyMass-Index (BMI) erfaßt, d. h. des Verhältnisses des Körpergewichts zum Quadrat der Körpergröße (11). Außerdem wurde der Broca-Index unter Verwendung folgender Formel errechnet:

$$
\frac{\text { Körpergewicht }(\mathrm{kg})}{\text { Körpergröße (cm) }-100}
$$

Sämtliche Rohdaten wurden auf Lochkarten übertragen und an der EDV-Anlage (Siemens 404/3) des Sonderforschungsbereichs 51 gespeichert und weiterverarbeitet.

Um einen Zusammenhang (Korrelation) zwischen den verschiedenen Meßwerten nachzuweisen, wurde die Methode der linearen Regressionsrechnung angewendet. Da dies angenähert normalyerteilte Werte voraussetzt, wurde zunächst die Verteilung der Meßwerte mit dem $\chi^{2}$-Anpassungstest untersucht. Als einziger Parameter wiesen die Triglyceride eine signifikante Abweichung von der Normalverteilung auf. Durch $\log _{10}$-Transformation konnten aber auch diese Werte in eine normalverteilte Form gebracht werden. Bei allen statistischen Parametern, in die Triglyceridwerte eingehen, wurden daher stets die dekadischen Logarithmen der Meßwerte zur Berechnung verwendet.

Zur Ausschaltung eventueller Scheinkorrelationen wurden partielle Korrelationskoeffizienten errechnet. Bei der partiellen Korrelation wird auf rechnerischem Weg der Einfluß einer dritten Größe auf zwei korrelierende Meßwerte ausgeschaltet (z. B. Alter auf BMI und Cholesterin) (12).

Zur Bestimmung der Mittelwerte und Referenzbereiche für Cholesterin und Triglyceride wurde das Kollektiv zunächst nach Geschlecht und Altersdekaden aufgeteilt. In einem ersten Durchlauf am Rechner wurden diejenigen Werte eliminiert, die in der jeweiligen Gruppe außerhalb des 2-s-Berèiches lagen. Die Prüfung durch den $x^{2}$-Anpassungstest ergab für die gesamten Cholesterinwerte des bereinigten Kollektives keine signifikante Abweichung von der Normalverteilung. Dasselbe traf auf die Triglyceridwerte nach deren Umwandlung in dekadische Logarithmen zu. Alle Triglyceridwerte wurden deshalb in Zehnerlogarithmen umgewandelt. Wegen der kleinen $\mathrm{n}-Z$ Zahlen ließen sich in den Unterkollektiven die Verteilungstypen nicht mehr erfassen. Als obere Normgrenze für das Cholesterin wurde die obere 2-s-Grenze gewählt. Für die Triglyceride hingegen wurde, wie auch von anderen Autoren $(13,14,15)$ die obere 1,7-sGrenze der logarithmierten Werte als obere Grenze des Referenzbereiches gewählt. Wegen der bei diesem Parameter sehr hohen Standardabweichung erschien uns die obere 2,0-s-Grenze als biologisch nicht sinnvoll.

Tab. 1. Konzentrationen von Cholesterin und Triglyceriden in Abhängigkeit von Alter und Geschlecht.

Mittelwerte und Referenzbereiche bei den Personen, deren Lipidwerte in einem ersten Rechengang innerhalb des 2-s-Bereiches lagen. $n=Z$ ahl aller Personen in der jeweiligen Altersklasse, deren Werte in den ersten Rechengang eingegeben wurden. Die

\begin{tabular}{|c|c|c|c|c|c|c|}
\hline & Alters- & $\mathbf{n}$ & Cholesterin & n Serum & Triglyceride & Serum \\
\hline & $\begin{array}{l}\text { gruppe } \\
\text { Jahre }\end{array}$ & & $\begin{array}{l}\overline{\mathrm{x}} \text {-Wert } \\
\mathrm{mmol} / \mathrm{l}(\mathrm{mg}\end{array}$ & $\begin{array}{l}\text { Untere und obere Grenze } \\
\text { des Referenzbereiches }\end{array}$ & $\begin{array}{l}\overline{\mathrm{x}} \text {-Wert } \\
\mathrm{mmol} / \mathrm{l}(\mathrm{mg}\end{array}$ & $\begin{array}{l}\text { Untere und obere Grenze } \\
\text { des Referenzbereiches }\end{array}$ \\
\hline Männer . & $\begin{array}{r}20-29 \\
30-39 \\
40-49 \\
50-59 \\
>59\end{array}$ & $\begin{array}{r}98 \\
186 \\
152 \\
91 \\
80\end{array}$ & $\begin{array}{l}4,53(175) \\
5,18(200) \\
5,28(204) \\
5,46(211) \\
5,70(220)\end{array}$ & $\begin{array}{r}2,95(114)-6,11(236) \\
3,40(131)-6,96(269) \\
3,34(129)-7,22(279) \\
3,96(154)-6,96(268) \\
3,82(147)-7,58(293)\end{array}$ & $\begin{array}{l}1,19(104) \\
1,43(125) \\
1,46(128) \\
1,35(118) \\
1,55(136)\end{array}$ & $\begin{array}{l}0,24(29)-2,14(188) \\
0,14(14)-2,71(236) \\
0,30(26)-2,62(230) \\
0,15(17)-2,51(219) \\
0,40(35)-2,70(237)\end{array}$ \\
\hline Frauen & $\begin{array}{r}20-29 \\
30-39 \\
40-49 \\
50-59 \\
>59\end{array}$ & $\begin{array}{l}42 \\
57 \\
42 \\
36 \\
30\end{array}$ & $\begin{array}{l}4,51(174) \\
4,99(193) \\
5,31(205) \\
5,72(221) \\
6,24(241)\end{array}$ & $\begin{array}{l}3,12(120)-5,91(228) \\
3,40(132)-6,58(254) \\
4,05(156)-6.57(254) \\
4,13(160)-7,31(282) \\
4,22(163)-8,26(319)\end{array}$ & $\begin{array}{l}0,98(86) \\
1,03(90) \\
1,12(98) \\
1,25(110) \\
1,39(122)\end{array}$ & $\begin{array}{l}0,45(39)-1,51(133) \\
0,77(33)-1,29(147) \\
0,16(17)-2,28(199) \\
0,56(48)-1.94(172) \\
0,64(57)-2,14(187)\end{array}$ \\
\hline
\end{tabular}
obere Grenze des Referenzbereiches ist für Serumcholesterin als $\bar{x}+2 \mathrm{~s}$, für Serumtriglyceride als $\bar{x}+1,7 \mathrm{~s}$ definiert. Bei der Triglyceridbestimmung wurde das freie Glycerin weder durch Messung noch durch Berechnung berïcksichtigt. 


\section{Ergebnisse}

Die Durchschnittswerte sowie die unteren und oberen Grenzen des Referenzbereiches für Cholesterin und Triglyceride in Abhängigkeit von Alter und Geschlecht sind in Tabelle 1 abgebildet. Die Häufigkeit der Hypercholesterinämie oder Hypertriglyceridämie in der untersuchten Bevölkerungsgruppe ist in Tabelle 2 angegeben. Als kombinierte Hyperlipidämie wurde die gleichzeitige Erhöhung von Cholesterin- und Triglyceridwerten bezeichnet. Die Häufigkeit aller Hyperlipidämien in der untersuchten Bevölkerungsgruppe lag bei über 14\%. Die häufigste Form der Hyperlipidämie war die Hypertriglyceridämie mit $7,3 \%$.

Tab. 2. Häufigkeit der Hyperlipidämien in der untersuchten Bevölkerungsgruppe.

Die Daten wurden an einer Bevölkerungsgruppe von 813 Personen erhoben. Als „Hypercholesterinämie" sind Werte außerhalb des 2-s-Bereiches, als, ,Hypertriglyceridämie" sind Werte außerhalb des 1,7-s-Bereiches definiert. Als ,,kombinierte Hyperlipidämie" sind solche Fälle bezeichnet, bei denen die Konzentrationen beider Serumlipide erhöht waren.

\begin{tabular}{lrc}
\hline & $\mathrm{n}$ & $\%$ \\
\hline Hypercholesterinämie & 37 & 4,54 \\
Hypertriglyceridämie & 59 & 7,25 \\
Kombinierte Hyperlipidämie & 20 & 2,46 \\
\hline & insgesamt 116 & 14,27
\end{tabular}

Die Cholesterinwerte der untersuchten Personen stiegen mit dem Alter an. Unterhalb des 45. Lebensjahres zeigten Frauen niedrigere Durchschnittswerte als Männer, während sie nach dem 45. Lebensjahr im Durchschnitt höhere Werte hatten (Abb. 1). Die Triglyceridwerte stiegen bei Männern mit dem Alter stetig an. Bei der weiblichen Bevölkerung war vom 20. bis 40 . Lebensjahr praktisch kein Anstieg der durchschnittlichen Triglyceridwerte zu beobachten, während sie nach dem 40. Lebensjahr anstiegen und nach dem 55. Lebensjahr mit denen der Männer gleichauf lagen (Abb. 2).

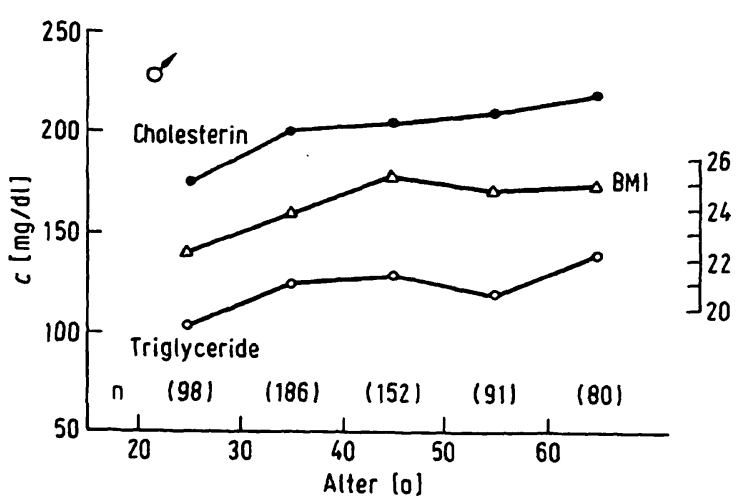

Abb. 1. Altersabhängigkeit der Mittelwerte von Serumcholesterin-, Serumtriglyceridkonzentrationen und BMI bei Männern. Die Lipidwerte im Serum basieren auf den Daten des "bereinigten" Kollektivs, der BMI wurde an Hand der Personen, bei denen Körpergewicht und Körperlänge bekannt war, berechnet. Bei der Triglyceridbestimmung wurde das freie Glycerin weder durch Messung noch durch Berechnung berücksichtigt.

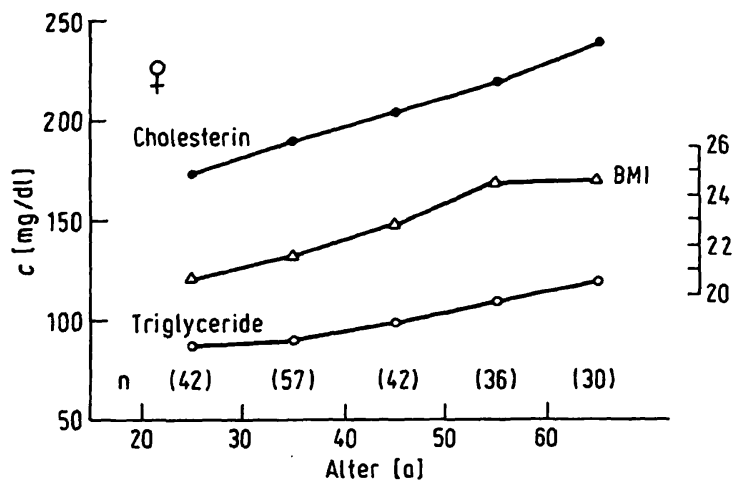

Abb. 2. Altersabhängigkeit der Mittelwerte von Cholesterin-, Triglyceridkonzentrationen und BMI bei Frauen. Die Lipidwerte im Serum basieren auf den Daten des ,bereinigten" Kollektivs, der BMI wurde an Hand der Personen, bei denen Körpergewicht und Körperlänge bekannt war, berechnet. Bei der Triglyceridbestimmung wurde das freie Glycerin weder durch Messung noch durch Berechnung berücksichtigt.

Tab. 3. Body Mass Index (BMI) und Relativgewicht nach Broca in Abhängigkeit von Alter und Geschlecht. Mittelwerte und obere 1,7-bzw. 2-s-Grenzen von Body Mass Index (BMI) und Relativgewicht nach Broca in den verschiedenen Mitterlwerte und obere $1,7-b$ w.

\begin{tabular}{|c|c|c|c|c|c|c|c|c|}
\hline & & Body & lex (BMI & $\times 10^{4}$ & & Broca & $\frac{K G}{K L-100}$ & \\
\hline & $\begin{array}{l}\text { Alters- } \\
\text { gruppe }\end{array}$ & $\mathbf{n}$ & $\overline{\mathbf{x}}$ & $\begin{array}{l}\text { Obere } \\
1,7-s- \\
\text { Grenze }\end{array}$ & $\begin{array}{l}\text { Obere } \\
2,0-s- \\
\text { Grenze }\end{array}$ & $\overline{\mathbf{x}}$ & $\begin{array}{l}\text { Obere } \\
1,7-s- \\
\text { Grenze }\end{array}$ & $\begin{array}{l}\text { Obere } \\
2,0-s- \\
\text { Grenze }\end{array}$ \\
\hline & Jahre & & & & & & & \\
\hline Männer & $\begin{array}{r}20-29 \\
30-39 \\
40-49 \\
50-59 \\
>59\end{array}$ & $\begin{array}{r}59 \\
126 \\
95 \\
56 \\
71\end{array}$ & $\begin{array}{l}22,47 \\
24,04 \\
25,46 \\
24,88 \\
25,23\end{array}$ & $\begin{array}{l}25,56 \\
27,66 \\
29,43 \\
28,47 \\
28,83\end{array}$ & $\begin{array}{l}26,10 \\
28,34 \\
30,12 \\
29,10 \\
29,47\end{array}$ & $\begin{array}{l}0,93 \\
0,99 \\
1,05 \\
1,03 \\
1,04\end{array}$ & $\begin{array}{l}1,06 \\
1,14 \\
1,21 \\
1,17 \\
1,19\end{array}$ & $\begin{array}{l}1,08 \\
1,17 \\
1,24 \\
1,20 \\
1,22\end{array}$ \\
\hline Frauen & $\begin{array}{r}20-29 \\
30-39 \\
40-49 \\
50-59 \\
>59\end{array}$ & $\begin{array}{l}30 \\
52 \\
26 \\
24 \\
29\end{array}$ & $\begin{array}{l}20,86 \\
21,78 \\
23,11 \\
24 ; 82 \\
24,94\end{array}$ & $\begin{array}{l}24,57 \\
24,78 \\
28,08 \\
29,39 \\
30,10\end{array}$ & $\begin{array}{l}25,22 \\
25,39 \\
28,96 \\
30,19 \\
31,04\end{array}$ & $\begin{array}{l}0,86 \\
0,88 \\
0,95 \\
1,02 \\
1,03\end{array}$ & $\begin{array}{l}1,01 \\
1,02 \\
1,16 \\
1,21 \\
1,24\end{array}$ & $\begin{array}{l}1,04 \\
1,05 \\
1,20 \\
1,25 \\
1,28 \\
\end{array}$ \\
\hline
\end{tabular}


Tabelle 3 enthält den BMI und das durch den BrocaIndex ermittelte Relativgewicht, getrennt nach Geschlecht und Altersdekaden. Auch hier wurden ausschließlich Werte des „, bereinigten“ Kollektives berücksichtigt.

Abbildung 1 und 2 zeigen die Altersabhängigkeit der Mittelwerte von Cholesterin, Triglyceriden und Körpergewicht bei Frauen und bei Männern. BMI, Relativgewicht nach Broca und die Serumlipide, insbesondere die Serumtriglyceride zeigten ein ähnliches Verhalten in Bezug auf das Lebensalter. Ob zwischen dem Körpergewicht und den Lipidwerten im Serum unabhängig vom Alter eine statistische Korrelation besteht, wurde im weiteren geprüft.

Zunächst wurden die Mittelwerte und Standardabweichungen aller nach Alter und Geschlecht korrigierten Lipidwerte im Serum berechnet (Tab. 4). Das Verteilungsmuster dieser beiden Parameter wurde durch den $\chi^{2}$-Anpassungstest geprüft. Die korrigierten Cholesterinwerte waren ebenso wie die korrigierten und logarithmierten Triglyceridwerte annähernd normal verteilt:

Tab. 4. Mittelwerte und obere Normalgrenze der alters- und geschlechtskorrigierten Cholesterin- und Triglyceridwerte.

Der Berechnung der aufgeführten Werte liegt die unter Methoden aufgefuihrte Formel zugrunde. Bei der Triglyceridbestimmung wurde das freie Glycerin weder durch Messung noch durch Berechnung berïcksichtigt.

\begin{tabular}{ll}
\hline Cholesterin & Triglyceride \\
$\mathrm{mmol} / \mathrm{l}(\mathrm{mg} / \mathrm{dl})$ & $\mathrm{mmol} / \mathrm{l}(\mathrm{mg} / \mathrm{dl})$ \\
\hline$\overline{\mathrm{x}}=5,31(205)$ & $\overline{\mathrm{x}}=1,20(105)$ \\
$\mathrm{s}= \pm 0,83( \pm 32)$ & $\mathrm{s}= \pm 0,71( \pm 62)$ \\
Obere Normgrenze & Obere Normgrenze \\
$=6,97(269)$ & $=2,41(211)$ \\
\hline
\end{tabular}

Dann wurde untersucht, ob bei übergewichtigen Patienten die Häufigkeit der Hyperlipidämie höher lag als bei dem Rest der Bevölkerungsgruppe. Wie aus Tabelle 5 zu sehen ist, wurde bei Patienten mit niedrigem Körpergewicht eine geringere Häufigkeit von Hyperlipidämien gefunden. Tabelle 6 zeigt die Mittelwẹrte und Standardabweichungen der nach Alter und Geschlecht korrigierten Lipide im Serum bei adipösen und nicht adipösen Patienten. Bei den adipösen Patienten lagen die Lipide im Serum im Durchschnitt höher. Weiterhin wurde die lineare Korrelation zwischen BMI und Lipidwerten im Serum geprüft. Diese Ergebnisse sind in Tabelle 7 angegeben.

Tab. 7. Lineare Korrelationskoeffizienten zwischen Cholesterin, Triglyceriden, BMI und prozentualem Übergewicht. Es wurden alle Personen, von denen Körperlänge und -gewicht bekannt waren, zugrunde gelegt $(n=586)$. Bei der Triglyceridbestimmung wurde das freie Glycerin weder durch Messung noch durch Berechnung berücksichtigt.

\begin{tabular}{lll}
\hline & $\mathrm{r}$ & $\mathrm{p}$ \\
\hline BMI - Cholesterin (korr) & 0,0662 & nicht signifik. \\
BMI - log Trigly ceride (korr) & 0,1979 & $<0,001$ \\
BMI - log Triglyceride & 0,2869 & $<0,001$ \\
BMI - Broca Index & 0,9832 & $<0,001$ \\
BMI - Lebensalter & 0,3246 & $<0,001$ \\
Lebensalter - Cholesterin & 0,3738 & $<0,001$ \\
Lebensalter - log Triglyceride & 0,1680 & $<0,001$ \\
\hline
\end{tabular}

Tab. 5. Häufigkeit von Hyperlipidämien in Abhängigkeit vom Körpergewicht.

Alle Personen, von denen Körpergewicht und Körperlänge bekannt waren $(n=586)$, wurden in fünf Gewichtsklassen aufgegliedert. In jeder Gewichtsklasse wurde die Häufigkeit von Hyperlipidämien (\%) ermittelt (Normgrenzen s. Tab. 1).

\begin{tabular}{lllllll}
\hline Gewichtsklasse & BMI & $<20,1$ & $20,1-23,0$ & $23,0-25,4$ & $25,4-28,0$ & $>28,0$ \\
& Broca & $<0,85$ & $0,85-0,95$ & $0,95-1,05$ & $1,05-1,15$ & $>1,15$ \\
& & $\mathrm{n}=65$ & $\mathrm{n}=134$ & $\mathrm{n}=190$ & $\mathrm{n}=110$ & $\mathrm{n}=69$ \\
\hline Hypercholesterinämie & & 1,54 & 1,49 & 2,63 & 5,45 & 5,80 \\
Hypertriglyceridämie & & 4,62 & 6,72 & 11,05 & 9,09 & 8,70 \\
Kombinierte Hyperlipidämie & & 1,54 & 0,75 & 2,63 & 4,55 & 2,90 \\
\hline
\end{tabular}

Tab. 6. Mittelwerte und Standardabweichungen der nach Alter und Geschlecht korrigierten Lipide im Serum bei Personen der verschiedenen Gewichtsklassen.

Es wurde die gleiche Einteilung in Gewichtsklassen vorgenommen, wie in Tabelle 5 angegeben. Wegen der Alters- und Geschlechtskorrektur siehe Tabelle 4.

\begin{tabular}{llllrrrrr}
\hline Gewichtsklasse & BMI & $<20,1$ & $20,1-23,0$ & $23,0-25,4$ & $25,4-28,0$ & $>28,0$ \\
& Broca & $<0,85$ & $0,85-0,95$ & $0,95-1,05$ & $1,05-1,15$ & $>1,15$ \\
\hline Cholesterin (korr) & $\bar{x}$ & $5,44(210)$ & $5,34(206)$ & $5,39(208)$ & $5,59(216)$ & 5,52 & $(213)$ \\
mmol/1 (mg/dl) & $\mathrm{s}$ & $\pm 0,78(30)$ & $\pm 0,85$ & $(33)$ & $\pm 0,98(38)$ & $\pm 1,01 \cdot(39)$ & $\pm 1,06$ & $(41)$ \\
Triglyceride (korr) & $\overline{\mathrm{x}}$ & $1,11(98)$ & $1,19(105)$ & $1,34(117)$ & $1,40(123)$ & $1,47(129)$ & $(1,4)$ \\
mmol/1 (mg/dl) & $\mathrm{s}$ & $\pm 0,02(1,48)$ & $\pm 0,019(1,58)$ & $\pm 0,18(1,58)$ & $\pm 0,019(1,66)$ & $\pm 0,018(1,55)$ \\
\hline
\end{tabular}


Zwischen BMI und Cholesterinwerten ergab sich keine positive Korrelation. Weiterhin wurde untersucht, ob die Lipidwerte unabhängig vom Alter mit dem BMI korrelieren. Dies geschah durch die Prüfung der partiellen Korrelation. Wie die Tabelle 8 zeigt, trifft die Hypothese für die Triglyceridwerte zu, für das Cholesterin hingegen nicht.

Aus der Beziehung zwischen Körpergewicht und Triglyceriden läßt sich eine Formel zur Berechnung der bei einem bestimmten Körpergewicht zu erwartenden Triglyceridkonzentration ableiten. Sie lautet bei Verwendung des Body Mass Index (BMI): Triglyceridkonzentration $(\mathrm{mg} / \mathrm{dl})=36,3 \times 10^{0.02 \times} \mathrm{BMI}$. Diese Beziehung läßt für eine übergewichtige Bevölkerungsgruppe mit einem durchschnittlichen BMI von 27.7 (Broca $+15 \%)$ und einer mittleren Triglyceridkonzentration von $130 \mathrm{mg} / \mathrm{dl}$ erwarten, daß sich bei Verringerung des mittleren Ubergewichts um $10 \mathrm{~kg}$ eine Triglyceridkonzentration von nunmehr $111 \mathrm{mg} / \mathrm{dl}$ einstellen würde. Wie Abbildung 3 zeigt, sind wegen der großen Streuung solche Aussagen für Einzelpersonen allerdings nicht zulässig.

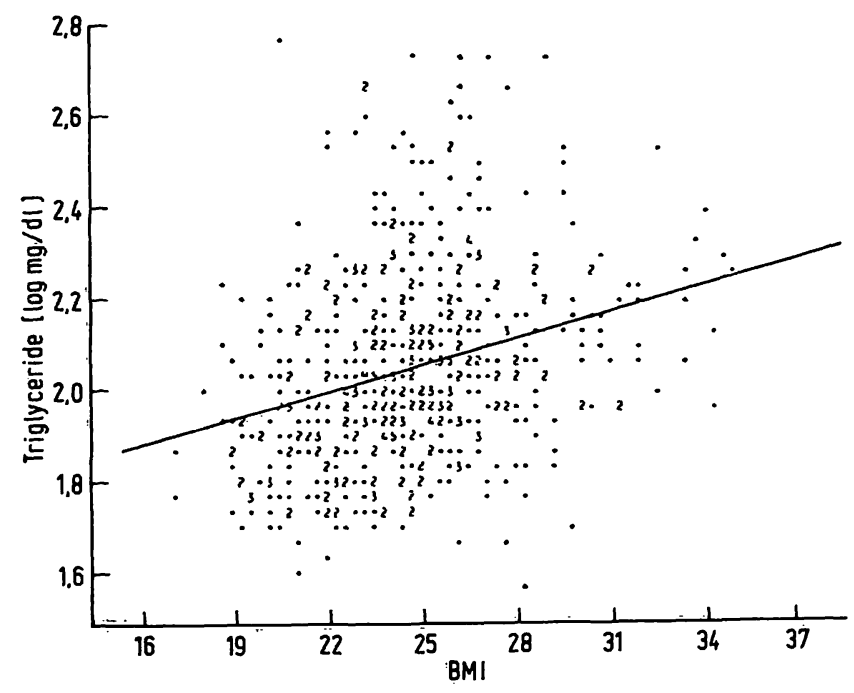

Abb. 3. Graphische Darstellung der Korrelation zwischen dem BMI und den logarithmierten Triglyceridwerten.

Die lineare Regression mit 567 Wertpaaren wurde geprüft. Gleichung der Regressionsgeraden:

$y=0,02 x+1,56$. Der Korrelationskoeffizient ( $r$ ) betrug $0,286(p<0,001)$.

Bei der Triglyceridbestimmung wurde das freie Glycerin weder durch Messung noch durch Berechnung berücksichtigt.

\section{Diskussion}

Cholesterin und Triglyceride sind Bestandteile sämtlicher Lipoproteine des Serums. In der Entstehung der Atherosklerose kommt den Lipoproteinen niedriger Dichte (LDL), die den größten Teil des Cholesterins transportieren, eine wichtige Bedeutung zu (16). Neuere, überwiegend epidemiologische Befunde sprechen für eine antiatherogene Wirkung der Lipopro-
Tab. 8. Korrelationskoeffizienten bei Prüfung der partiellen Korrelation zwischen BMI und Lipiden im Serum. Es wurden alle Personen, von denen Körperlänge und Körpergewicht bekannt waren, zugrunde gelegt $(n=586)$.

Bei der Triglyceridbestimmung wurde das freie Glycerin weder durch Messung noch durch Berechnung berücksichtigt.

\begin{tabular}{lll}
\hline & $\mathrm{r}$ & $\mathrm{p}$ \\
\hline $\begin{array}{l}\text { BMI - log Triglyceride } \\
\text { (unabhängig vom Alter) }\end{array}$ & 0,249 & $<0,001$ \\
$\begin{array}{l}\text { BMI - Cholesterin } \\
\text { (unabhängig vom Alter) }\end{array}$ & 0,097 & $\begin{array}{l}\text { nicht } \\
\text { signifikant }\end{array}$ \\
\hline
\end{tabular}

teine mit hoher Dichte (HDL) (17). Dieser Zusammenhang ist aber experimentell noch nicht ausreichend bewiesen (18).

Die Bestimmung von Gesamtcholesterin und Nüchterntriglyceriden ist das Basisprogramm der Lipiddiagnostik für die Erfassung des coronaren Risikos. Voraussetzung für die Abschätzung des coronaren Risikos ist die Ermittlung von Referenz- oder sogenannten Normalwertbereichen für die Lipidkonzentrationen. Es muß aber zwischen dem statistisch gefundenen Normalwertbereich in einer Bevölkerungsgruppe und dem biologisch „Gesunden" unterschieden werden. Auch innerhalb des Referenzwertbereiches nimmt das Coronarrisiko mit der Höhe des Cholesterins zu (16).

Lipidwerte im Serum werden von genetischen Faktoren, Ernährungsgewơhnheiten und Krankheiten beeinflußt. Beim Vergleich der Lipidwerte verschiedener Bevölkerungsgruppen müssen Auswahl der Personen und die Anwendung unterschiedlicher Methoden der Bestimmung der Lipide im Serum sowie der Auswertung der Daten berïcksichtigt werden.

Die von uns ermittelten Durchschnittswerte und Normalwertbereiche für das Cholesterin stimmen mit Ergebnissen aus den USA gut überein $(16,17,18)$. Ähnliche Resultate wurden auch von deutschen Autoren veröffentlicht (18, 19, 20). Allerdings ist ein Vergleich mit diesen und ähnlichen Arbeiten aufgrund einer anderen Einteilung der Altersklassen $(19,20,21)$ oder der Verwendung nichtenzymatischer Bestimmungsmethoden (21) nur mit Einschränkung möglich. Mit der vollenzymatischen Bestimmungsmethode wurden die Cholesterinwerte eines alle Altersklassen über 20 Jahre umfassenden Kollektivs mindestens in Mitteleuropa bisher nicht erfaßt. Die Cholesterinkonzentrationen der von uns untersuchten männlichen Bevölkerung stimmen mit den Ergebnissen der "Seven countries study" (27) gut überein. Sie liegen niedriger als $z$. B. in Finnland, höher als in südeuropäischen Ländern. Diese unterschiedlichen Cholesterinwerte werden auf die Ernährung, vielleicht auch auf genetische Faktoren zurückgefuihrt.

Einige Autoren berichten von einem Abfall der durchschnittlichen Cholesterinkonzentration bei Männern über 55 Jahren $(13,15,19)$. Diese Beobachtung konnte 
in unserer, wie auch in anderen Bevölkerungsgruppen nicht bestätigt werden $(14,21)$.

Die Triglyceridwerte stimmen mit den Ergebnissen anderer Autoren weitgehend überein $(13,14,15,19$, 21, 22). Die Durchschnittswerte und Normalgrenzen der Serumlipide der Münchner Bevölkerungsgruppe zeigen einen stetigen Anstieg mit dem Lebensalter. Dieser Befund deckt sich mit der Literatur $(13,16$, 17, 19, 23). Es ist deshalb notwendig, bei der Entscheidung, ob ein Lipidwert als pathologisch anzusehen ist, das Lebensalter ebenso zu berücksichtigen, wie die Zugehörigkeit zu einer bestimmten Bevölkerungsgruppe.

Wenn man sämtliche Cholesterinwerte oberhalb von 260 $\mathrm{mg} / \mathrm{dl}$ Serum als pathologisch betrachtet, so finden sich in der männlichen Bevölkerung unterhalb von 45 Jahren nur 7,4\% Hypercholesterinämien, während im Rest der untersuchten Bevölkerung die Häufigkeit etwa $15,7 \%$ beträgt. $\mathrm{Da}$ aber die frühzeitige Therapie der Hyperlipidämien als Prävention der coronaren Herzkrankheit erstrebenswert ist, kommt einer genauen Abgrenzung von normalen zu pathologischen Werten gerade beim jüngeren Patienten eine große Bedeutung zu. Die Altersabhängigkeit der Cholesterin- und Triglyceridwerte sollte deshalb beachtet werden.

In der von uns untersuchten Bevölkerungsgruppe wurde keine positive Korrelation zwischen BMI und Cholesterinwerten ermittelt (Tab. 7). In der Tecumseh-Studie zeigte sich eine gering signifikante Korrelation zwischen Hautfaltendicke oder relativem Gewicht und der Cholesterinkonzentration (24). Ähnlich geringe Korrelationen fanden auch andere Autoren (25). Keine Korrelation konnte von Albrink \& Meigs (26) sowie Gibson (27) gefunden werden. Wie aus unseren Daten hervorgeht, steigen Cholesterinkonzentration und BMI mit dem Alter an. Eine vom Alter unabhängige Korrelation zwischen ihnen kommt jedoch nicht zustande (Tab. 8). Möglicherweise sind dadurch die widersprüchlichen Befunde in der Literatur über den Zusammenhang zwischen Körpergewicht und Cholesterinkonzentration im Serum zu erklären.

Zwischen BMI und Triglyceriden im Serum würde eine positive Korrelation gefunden. Dieses Ergebnis deckt sich mit denen der Literatur $(17,26,28,29)$. Die Kurven der Altersabhängigkeit von Triglyceriden und BMI verlaufen parallel (Abb. 1 und 2). Die positive Korrelation zwischen BMI und Triglyceriden besteht aber auch unabhängig vom Alter. In Übereinstimmung mit anderen Autoren (30) wird eine größere Häufigkeit der Hypertriglyceridämie unter den stark adipösen Personen gefunden. Die Hypertriglyceridämie spricht sehr gut auf Gewichtsreduktion an $(31,32)$, weil dadurch die Produktion von triglyceridreichen Lipoproteinen erniedrigt wird (33). In der Therapie der Hypertriglyceridämie sollte deshalb bei Bestehen eines Übergewichts als erste therapeutische Maßnahme eine Gewichtsreduktion angestrebt werden.

\section{Danksagung}

Wir danken Frau $R$. Fischer für die sorgfältige technische Assistenz und Frl. B. Schürmann für die wirkungsvolle organisatorische Hilfe.

\section{Literatur}

1. Epstein, F. J., Ostrander, L. D. \& Johnson, B. C. (1965) Ann. Intern. Med. 62, 1170-1187.

2. Kannel, W. B., Castelli, W. P., Gordon, R. \& McNamara, P. M. (1971) Ann. Intern. Med. 74, 1-12

3. Carlson, L. A., Böttiger, L. E. \& Anfeldt, P. E. (1979) Acta Med. Scand. 206, 351-360.

4. Logan, R. L., Thompson, M., Riemersa, R. A., Oliver, M. F., Olsson, A. G., Rössner, S., Callmer, E., Walldius, G., Kaijser, L., Carlson, L. A., Lockerbie, L. \& Lutz, W. (1978) Lancet $I$ 949-955.

5. Gordon, T. \& Kannel, W. B. (1973) Geriatrics 28, 80-85.

6. Keys, A., Aravanis, C., Blackburn, H., Van Buchem, F. S., Buzina, R., Djrodjenivic, B. S., Fidanza, F., Karvonen, M. J., Menotti, A., Puddu, V. \& Taylor, H. L. (1972) Ann. Intern. Med. 77, 15-27.

7. Weber, G., Fabbrini, P., Resi, L., Jones, R., Vesselinovitch, D. \& Wissler, R. W. (1977) Artherosclerosis 26, 535-547.

8. Barndt, R. Jr., Blankenhom, D. H., Crawford, D. W. \& Brooks, S. H. (1977) Ann. Intern. Med. 86, 139-146.

9. Röschlau, P., Bernt, W. \& Gruber, W. (1974) Z. Klin. Chem. $12,226-227$.

10. Eggstein, M. \& Kreutz, F. H. (1966) Klin. Wochenschr. 44, 262-267.

11. Keys, A., Fidanza, F., Karvonen, M. J., Kimura, N. \& Taylor, H. L. (1972) J. Chron. Dis. 25, 329-343.

12. Sachs, L. (1972) Statistik, Springer-Verlag, 3. Aufl.
13. Goldstein, J. L., Hazzard, W. R., Schrott, H. G., Biermann, E. L. \& Motulsky, A. G. (1973) J. Clin. Invest. 52, 1544-1568.

14. Nikkilä, E. A. \& Aro, A. (1975) Postgr. Med. J. 51, 32-35.

15. Fredrickson, D. S., Goldstein, J. L. \& Brown, M. S. (1978) The Metabolic Basis of inherited Diseases. McGrawHill, Book Company S. 606.

16. Gotto, A. M. \& Jackson, R. L. (1978) Atheroscler. Rev. 3, 231.

17. Levy, R. I. (1978) Lipids 13, 911-913.

18. Keys, A. C. (1980) Lancet ii, 603-606.

19. Brown, D. F. \& Daudiss, K. (1973) Circulation 47, 558-566.

20. Woods, P. D. S., Stern, M. P., Silvers, A., Reaven, G. M. \& von der Groeben, J. (1972) Circulation 65, 114-126.

21. Johnson, D. C., Epstein, F. H. \& Kjelsberg, M. O. (1965) J. Chron. Dis. $18,147-160$.

22. Weißhaar, D. (1975) Med. Welt 26, 940-944.

23. Kattermann, R., Köhring, B. Brunne, B. (1976) Deut. Med. Wochenschr. 25, 953-957.

24. Hofmeister, H. (1978) Epidemiologische Felduntersuchungen in Hessen. Dietrich Reimer Verlag.

25. Keys, A. (1970) Circulation 41 (Suppl. 1), 1-211.

26. Lidgren, F. T., Adamsoñ, G. L., Jemson, L. C. \& Wood, P. D. (1975) Lipids 12, 750-756.

27. Montoye, J. Y., Epstein, F. H. \& Kjelsberg, M., O. (1966) Amer. J. Clin. Nutri. 18, 387-406. 
28. Hollister, L. E, Overall, J. E. \& Snow, H. L. (1976), Amer. J. Clin. Nutr. 20, 777-782.

29. Albrink, M. J. \& Meigs, J. W. (1964) Amer. J. Clin. Nutr. $15,255-260$.

30. Gibson, T. C., Horton, E. S. \& Whorton, E. B. (1975) Amer. J. Clin. Nutr. 28, 1387-1394.

31. Bagdade, J. D., Bierman, E. L. \& Porte, D. (1971), Diabetes 20, 664-672.

32. Sailer, S., Sandhofer, F. \& Braunsteiner, H. (1966) Metabolism $15,135-137$.
33. Albrink, J. M. \& Man, E. B. (1959) Arch. Intern. Med. 103, 4-8.

34. Moore, N. S., Young, C. M. \& Maynard, L. A. (1955) Amer. J. Med. 17, 348-354.

35. Moore, N. S., Fryer, J. H., Young, C. M. \& Maynard, L. A. (1955) Amer. J. Clin. Nutr. 3, 397-402.

36. Olefsky, J., Reaven, G. M. \& Farquhar, J. W. (1974) J. Clin. Invest. 53, 64-76.
Dr. med. K. Henze Medizinische Poliklinik der Universität

Pettenkoferstraße 8a

D-8000 München 2 
